\title{
LETTER
}

\section{A promising new therapy may assist efforts to combat ICU-acquired weakness}

\author{
Sarah Bain ${ }^{* *}$ and Meagan Littlepage ${ }^{2}$
}

In previous issues of Critical Care and other publications, authors have characterized emerging therapies such as neuromuscular electrical stimulation (NMES) and functional electrical stimulation (FES)-cycling as promising tools to help prevent the onset of ICU-acquired weakness [1-3]. Despite compelling preliminary data, practical challenges with these technologies have limited their use. For example, there are documented limitations in NMES efficacy in patients with obesity and peripheral limb edema [3], and one study demonstrated treatment failures in 50\% of patients, even in a non-obese cohort [4]. FES-cycling appears to perform more consistently (80\% success) [2], but is associated with relatively long set-up times (15 to $30 \mathrm{mi}-$ nutes for a two-person team [2]) that make widespread use challenging when available resources are limited.

Motivated by recently presented research [5], we've deployed a newly available variation of NMES, thermalenhanced muscle stimulation (supplied via the Niveus Medical System 110), in our critical care unit. This technique combines local hypothermia with electrical energy delivery in a manner that was shown to increase effectiveness and improve comfort [5]. While not intended to replace early team-driven mobilization, we hoped that this new method could provide more consistent efficacy than traditional NMES and facilitate our early mobility initiatives without adding significant burden to our staff.

Our experience led to several notable findings. Though our results are anecdotal, treated patients have consistently exceeded our expectations with regard to both strength and level of function following extended periods of immobilization. Additionally, we estimate that successful treatments have occurred in greater than $95 \%$ of sessions, which is substantially higher than reports of traditional NMES effectiveness in this cohort. To date, meaningful muscle contractions have been documented in patients with a body mass index as high as 42 and in patients with significant edema (for example, in a patient with a 21

\footnotetext{
* Correspondence: SarahBainECH@gmail.com

'Department of Anesthesia, El Camino Hospital, 2500 Grant Road, Mountain View, CA 94040, USA
}

kilogram weight gain from admission). With regard to patient comfort, it is common to see alert patients calmly experiencing quadriceps contractions strong enough to elevate heels from the bed bilaterally. Lastly, we found that bedside nursing can set up treatment in less than 5 minutes, minimizing distraction from other aspects of patient care and early rehabilitation.

Our experience using thermal-enhanced muscle stimulation has been overwhelmingly positive. We believe this technique is simple to deploy and could be a strong complement to other activities included in the $\mathrm{ABCDE}$ bundle. We hope our experiences will be informative to others when developing their own strategies for earlier rehabilitation in critically ill patients.

\section{Abbreviations}

FES: Functional electrical stimulation; NMES: Neuromuscular electrical stimulation.

\section{Competing interests}

The authors declare that they have no competing interests.

\section{Author details}

'Department of Anesthesia, El Camino Hospital, 2500 Grant Road, Mountain View, CA 94040, USA. ²Department of Physical Medicine and Rehabilitation, El Camino Hospital, 2500 Grant Road, Mountain View, CA 94040, USA.

Published online: 23 October 2014

\section{References}

1. Ali NA: Have we found the prevention for intensive care unit-acquired paresis? Crit Care 2010, 14:160.

2. Parry SM, Berney S, Warrillow S, El-Ansary D, Bryant AL, Hart N, Puthucheary Z, Koopman R, Denehy L: Functional electrical stimulation with cycling in the critically ill: a pilot case-matched control study. J Crit Care 2014, 29:695.e1-695.e7.

3. Callahan LA, Supinski GS: Prevention and treatment of ICU-acquired weakness: is there a stimulating answer? Crit Care Med 2013, 41:2457-2458.

4. Gosselink R, Segers J, Hermans G, Bruyninckx F, Meyfroidt G, Langer D: Neuromuscular electrical stimulation in patients admitted to the intensive care unit: a feasibility study. Am J Respir Crit Care Med 2013, 187:A3627.

5. Flaugher M, Callaghan M, Nouri B: Performance of thermal-enhanced electrical muscle stimulation in challenging subjects: a randomized controlled trial. Am J Respir Crit Care Med 2014, 189:A6664.

doi:10.1186/s13054-014-0573-2

Cite this article as: Bain and Littlepage: A promising new therapy may assist efforts to combat ICU-acquired weakness. Critical Care 2014 18:573.
C Biomed Central

(c) 2014 Bain and Littlepage; licensee BioMed Central Ltd. This is an Open Access article distributed under the terms of the Creative Commons Attribution License (http://creativecommons.org/licenses/by/4.0), which permits unrestricted use, distribution, and reproduction in any medium, provided the original work is properly credited. The Creative Commons Public Domain Dedication waiver (http://creativecommons.org/publicdomain/zero/1.0/) applies to the data made available in this article, unless otherwise stated. 